\title{
On submanifolds of Kenmotsu manifold with Torqued vector field
}

\author{
Halil İbrahim Yoldaş* (D), Şemsi Eken Meriç (D), Erol Yaşar (D) \\ Department of Mathematics, Faculty of Science and Arts, Mersin University, 33343 Mersin, Turkey
}

\begin{abstract}
In this paper, we consider the submanifold $M$ of a Kenmotsu manifold $\tilde{M}$ endowed with torqued vector field $\mathcal{T}$. Also, we study the submanifold $M$ admitting a Ricci soliton of both Kenmotsu manifold $\tilde{M}$ and Kenmotsu space form $\tilde{M}(c)$. Indeed, we provide some necessary conditions for which such a submanifold $M$ is an $\eta$-Einstein. We have presented some related results and classified. Finally, we obtain an important characterization which classifies the submanifold $M$ admitting a Ricci soliton of Kenmotsu space form $\tilde{M}(c)$.
\end{abstract}

Mathematics Subject Classification (2010). 53C25, 53C40.

Keywords. Kenmotsu manifold, Ricci soliton, Torqued vector field.

\section{Introduction}

Hamilton introduced the concept of Ricci soliton, which is a natural generalization of Einstein manifold, in 1982 [11]. This notion actually corresponds to the self-similar solution of Hamilton's Ricci flow: $\frac{\partial}{\partial t} g=-2 \tilde{S}$, viewed as a dynamical system on the space of Riemannian metrics modulo diffeomorphims and scaling, (for details, see [12]).

In the framework of the contact geometry, Sharma started the studying of the problem of the Ricci solitons in K-contact manifolds in [18]. After this work, Ricci solitons have been investiaged in some different classes of contact geometry. For instance, it is proved by Ghosh that the constant curvature of a Kenmotsu 3-manifold as Ricci soliton is -1 in [10]. Then, Perktaş and Keleş proved that if a 3-dimensional normal almost paracontact metric manifold admits a Ricci soliton then it is shrinking in [17]. For more details, see $([1,2,8,9,16,19,21])$.

Consider the following equation on a Riemannian manifold $(\tilde{M}, g)$

$$
\left(£_{V} g\right)(X, Y)+2 \tilde{S}(X, Y)+2 \lambda g(X, Y)=0,
$$

where $£_{V} g$ is the Lie-derivative of the metric tensor $g$ in the direction vector field $V, \tilde{S}$ is the Ricci tensor of $\tilde{M}$ and $\lambda$ is a constant. $(\tilde{M}, g)$ is called a Ricci soliton if the equation (1.1) holds for vector fields $X, Y$ on $\tilde{M}$. The vector field $V$ is called the potential field of Ricci soliton $(\tilde{M}, g)$. If $£_{V} g=\rho g$, then potential field $V$ is said to be conformal Killing,

*Corresponding Author.

Email addresses: hibrahimyoldas@gmail.com(H. İ. Yoldaş), semsieken@hotmail.com(Ş. E. Meriç), eroly69@gmail.com(E. Yaşar)

Received: 06.11.2018; Accepted: 17.04.2019 
where $\rho$ is a function. If $\rho$ vanishes identically, then $V$ is said to be Killing. Also, if $V$ is zero or Killing in (1.1), then the Ricci soliton is called trivial and in this case, the metric is an Einstein. In addition, a Ricci soliton is called a gradient if the potential field $V$ is the gradient of a potential function $-f$ (i.e., $V=-\nabla f$ ) and is called shrinking, steady or expanding depending on $\lambda<0, \lambda=0$ or $\lambda>0$, respectively.

On the other hand, Riemannian manifolds which admit torqued vector fields (as a combination of concurrent and recurrent vector fields) were first defined by Chen in [6]. According to this definition, a nowhere zero vector field $\mathcal{T}$ on a Riemannian manifold $(\tilde{M}, g)$ is called torqued vector field, if it satisfies the following two conditions

$$
\tilde{\nabla}_{X} \mathcal{T}=f X+\alpha(X) \mathcal{T} \quad \text { and } \quad \alpha(\mathcal{T})=0,
$$

where $\tilde{\nabla}$ is the Levi-Civita connection on $\tilde{M}$, for any $X \in \Gamma(T \tilde{M})$. The function $f$ is called the torqued function and $1-$ form $\alpha$ is called the torqued form of $\mathcal{T}$. Here, Chen characterized rectifying submanifolds for a Riemannian manifold endowed with torqued vector field in [6]. Then, Chen proved that every Ricci soliton with torqued potential field is an almost quasi-Einstein under some conditions (see [7]).

The paper is organized as follows:

In Section 2, we recall some basic notions which are going to be needed.

In Section 3, we consider the submanifold $M$ of Kenmotsu manifold $\tilde{M}$ endowed with a torqued vector field $\mathcal{T}$ and find that the characteristic vector field $\xi$ of $\tilde{M}$ is never torqued on the ambient space $\tilde{M}$. Also, we give a necessary and sufficient condition for which the tangential part $\mathcal{T}^{\top}$ of $\mathcal{T}$ is torse-forming on $M$.

In Section 4, we deal with Kenmotsu space form $\tilde{M}(c)$ endowed with a torqued vector field $\mathcal{T}$ and give some characterizations on a submanifold admitting a Ricci soliton of $\tilde{M}(c)$.

The last section is devoted to conclusion. Here, we present our results which are obtained in this paper.

\section{Preliminaries}

In this section, we shall review some basic definitions and formulas of almost contact metric manifolds from $[3,4,15,20]$ and [22].

Let $\tilde{M}$ be an $(2 n+1)$-dimensional almost contact metric manifold with an almost contact metric structure $(\varphi, \xi, \eta, g)$ such that $\varphi$ is a tensor field of type $(1,1), \xi$ is a vector field (called the characteristic vector field) of type $(0,1), 1$ - form $\eta$ is a tensor field of type $(1,0)$ on $\tilde{M}$ and the Riemannian metric $g$ satisfies the following relations:

$$
\varphi^{2} X=-X+\eta(X) \xi, \quad \eta(\xi)=1, \quad \varphi \xi=0, \quad \eta \circ \varphi=0, \quad \eta(X)=g(X, \xi)
$$

and

$$
g(\varphi X, \varphi Y)=g(X, Y)-\eta(X) \eta(Y), \quad g(\varphi X, Y)=-g(X, \varphi Y)
$$

for any $X, Y \in \Gamma(T \tilde{M})$.

If the following condition is satisfied for an almost contact metric manifold $(\tilde{M}, \varphi, \xi, \eta, g)$, then it is called a Kenmotsu manifold

$$
\left(\tilde{\nabla}_{X} \varphi\right) Y=g(\varphi X, Y) \xi-\eta(Y) \varphi X
$$

where $\tilde{\nabla}$ is the Levi-Civita connection on $\tilde{M}$, for any $X, Y \in \Gamma(T \tilde{M})$. From (2.3), for a Kenmotsu manifold we also have

$$
\tilde{\nabla}_{X} \xi=X-\eta(X) \xi
$$


On the other hand, a Kenmotsu manifold $\tilde{M}$ with constant $\varphi$-sectional curvature $c$ is said to be a Kenmotsu space form and it is denoted by $\tilde{M}(c)$. The curvature tensor $\tilde{R}$ of a Kenmotsu space form is given by

$$
\begin{aligned}
\tilde{R}(X, Y) Z= & \frac{c-3}{4}\{g(Y, Z) X-g(X, Z) Y\} \\
& +\frac{c+1}{4}\{[\eta(X) Y-\eta(Y) X] \eta(Z) \\
& +[g(X, Z) \eta(Y)-g(Y, Z) \eta(X)] \xi \\
& +g(\varphi Y, Z) \varphi X-g(\varphi X, Z) \varphi Y-2 g(\varphi X, Y) \varphi Z\}
\end{aligned}
$$

for any $X, Y, Z \in \Gamma(T \tilde{M})$.

Let $M$ be isometrically immersed submanifold of Kenmotsu manifold $\tilde{M}$. For any $X, Y \in \Gamma(T M)$, we have

$$
\tilde{\nabla}_{X} Y=\nabla_{X} Y+h(X, Y)
$$

where $\tilde{\nabla}$ and $\nabla$ stand for the Levi-Civita connections on $\tilde{M}$ and $M$, respectively. Then, the equality (2.6) is called the Gauss formula and $h$ is called the second fundamental form of $M$. Also, if the second fundamental form $h$ vanishes identically in (2.6), then the submanifold $M$ is called totally geodesic. Similarly, one has

$$
\tilde{\nabla}_{U} V=-A_{V} U+\nabla_{U}^{\perp} V
$$

where $A_{V}$ and $\nabla^{\perp}$ denote the shape operator and the normal connection of $M$ in the ambient space $\tilde{M}$, respectively, for any $U \in \Gamma(T M)$ and $V \in \Gamma\left(T M^{\perp}\right)$. Using (2.4) and (2.6), it follows that

$$
\begin{aligned}
& \nabla_{X} \xi=X-\eta(X) \xi, \\
& h(X, \xi)=0,
\end{aligned}
$$

where $\nabla$ is the Levi-Civita connection of $M$.

Also, it is well known that the relation between second fundamental form $h$ and the shape operator $A_{V}$ are related by

$$
g\left(A_{V} X, Y\right)=g(h(X, Y), V)
$$

for any $X, Y \in \Gamma(T M)$. Here, we denote by the same symbol $g$ the Riemannian metric induced by $g$ on $\tilde{M}$.

The equation of Gauss is given by

$$
\begin{aligned}
g(R(X, Y) Z, W)= & g(\tilde{R}(X, Y) Z, W)+g(h(X, W), h(Y, Z)) \\
& -g(h(X, Z), h(Y, W))
\end{aligned}
$$

for any $X, Y, Z, W \in \Gamma(T M)$.

We denote by $H$ the mean curvature vector, that is,

$$
H(p)=\frac{1}{n} \sum_{i=1}^{n} h\left(e_{i}, e_{i}\right)
$$

where $\left\{e_{1}, e_{2}, \ldots, e_{n}=\xi\right\}$ is an orthonormal basis of the tangent space $T_{p} M, p \in M$. As it is known, $M$ is called minimal if $H$ vanishes identically.

The submanifold $M$ is $\omega$-umbilical with respect to a normal vector field $\omega$ if its shape operator satisfies $A_{\omega}=\mu I$, where $\mu$ is a function on $M$ and $I$ is the identity map.

Furthermore, the submanifold $M$ is said to be totally umbilical if and only if one has

$$
h(X, Y)=g(X, Y) H
$$


for any $X, Y \in \Gamma(T M)$, where $h$ and $H$ denote the second fundamental form and the mean curvature vector, respectively.

The scalar curvature $r$ of $(M, g)$ is defined by

$$
r=\sum_{i=1}^{n} S\left(e_{i}, e_{i}\right)
$$

where $\left\{e_{1}, e_{2}, \ldots, e_{n}=\xi\right\}$ is an orthonormal frame of $T M$ and $S$ is the Ricci tensor of $M$.

Now, we recall some definitions from $([7,14,22])$, as follows:

A Riemannian manifold $(\tilde{M}, g)$ is called $\eta$-Einstein if there exists two real constants $a$ and $b$ such that the Ricci curvature tensor field $\tilde{S}$ satisfies

$$
\tilde{S}(X, Y)=a g(X, Y)+b \eta(X) \eta(Y)
$$

for any $X, Y \in \Gamma(T \tilde{M})$. If the constant $b$ is equal to zero, then $\tilde{M}$ becomes an Einstein.

The Ricci tensor $\tilde{S}$ of a Kenmotsu manifold $(\tilde{M}, g)$ is called $\eta$-parallel if it satisfies

$$
\left(\tilde{\nabla}_{X} \tilde{S}\right)(\varphi Y, \varphi Z)=0
$$

such that

$$
\left(\tilde{\nabla}_{X} \tilde{S}\right)(\varphi Y, \varphi Z)=\tilde{\nabla}_{X} \tilde{S}(\varphi Y, \varphi Z)-\tilde{S}\left(\tilde{\nabla}_{X} \varphi Y, \varphi Z\right)-\tilde{S}\left(\varphi Y, \tilde{\nabla}_{X} \varphi Z\right)
$$

for any $X, Y, Z \in \Gamma(T \tilde{M})$.

A vector field $v$ on a Riemannian manifold $(\tilde{M}, g)$ is called torse-forming if it satisfies

$$
\tilde{\nabla}_{X} v=f X+\alpha(X) v
$$

where $f$ is a function, $\alpha$ is a $1-$ form and $\tilde{\nabla}$ is the Levi-Civita connection on $\tilde{M}$, for any $X \in \Gamma(T \tilde{M})$. The 1 -form $\alpha$ is called the generating form and the function $f$ is called the conformal scalar of $v$.

If the $1-$ form $\alpha$ in (2.13) vanishes identically, then the vector field $v$ is called concircular [5]. If $f=1$ and $\alpha=0$, then the vector field $v$ is called concurrent [23]. The vector field $v$ is called recurrent if it satisfies (2.13) with $f=0$. Also, if $f=\alpha=0$, the vector field $v$ in (2.13) is called parallel.

Let $\tilde{M}$ be a Kenmotsu manifold endowed with a torqued vector field $\mathcal{T}$ and $\phi: M \rightarrow \tilde{M}$ be an isometric immersion. Then, we get

$$
\mathcal{T}=\mathcal{T}^{\top}+\mathcal{T}^{\perp}
$$

where $\mathcal{T}^{\top}$ and $\mathcal{T}^{\perp}$ the tangential and normal components of $\mathcal{T}$ on $\tilde{M}$, respectively.

\section{The submanifolds admitting Ricci soliton of Kenmotsu manifolds}

In this section, we deal with the submanifold $M$ of Kenmotsu manifold $\tilde{M}$ endowed with torqued vector field $\mathcal{T}$.

From now on, we make the following:

Assumption. Throughout the paper, we suppose that the characteristic vector field $\xi$ is tangent to $M$.

Theorem 3.1. Let $\tilde{M}$ be a Kenmotsu manifold endowed with a torqued vector field $\mathcal{T}$. Then, the characteristic vector field $\xi$ is never torqued vector field on $\tilde{M}$. 
Proof. Since $\mathcal{T}$ is a torqued vector field on $\tilde{M}$, then we have

$$
\tilde{\nabla}_{X} \mathcal{T}=f X+\alpha(X) \mathcal{T} \quad \text { and } \quad \alpha(\mathcal{T})=0,
$$

where $\tilde{\nabla}$ stands for the Levi-Civita connection on $\tilde{M}$, for any $X \in \Gamma(T \tilde{M})$.

Suppose that $\xi$ is a torqued vector field on $\tilde{M}$. Using $\xi$ instead of $\mathcal{T}$ in equation (3.1), one has

$$
\tilde{\nabla}_{X} \xi=f X+\alpha(X) \xi \quad \text { and } \quad \alpha(\xi)=0 .
$$

Also, taking the inner product of (3.2) with $\xi$, we have

$$
\alpha(X)=-f \eta(X) .
$$

Therefore, the equation (3.2) reduces to

$$
\nabla_{X} \xi=f(X-\eta(X) \xi) .
$$

It follows from (2.8) and (3.3),

$$
f=1 \quad \text { and } \quad \alpha(X)=-\eta(X)
$$

are found.

On the other hand, if we take the characteristic vector field $X=\xi$ in (3.4), then we find

$$
\alpha(\xi)=-1
$$

which is a contradiction. Hence, $\xi$ is never torqued vector field on Kenmotsu manifold $\tilde{M}$.

The next example supports Theorem 3.1, as follows:

Example 3.2. ([13]). We consider the three-dimensional Riemannian manifold

$$
\tilde{M}=\left\{(x, y, z) \in \mathbb{R}^{3},(x, y, z) \neq(0,0,0)\right\},
$$

and the linearly independent vector fields

$$
e_{1}=z \frac{\partial}{\partial x}, \quad e_{2}=z \frac{\partial}{\partial y}, \quad e_{3}=-z \frac{\partial}{\partial z},
$$

where $(x, y, z)$ are the Cartesian coordinates in $\mathbb{R}^{3}$. Let $g$ be the Riemannian metric defined by

$$
\begin{aligned}
& g\left(e_{i}, e_{i}\right)=1 \\
& g\left(e_{i}, e_{j}\right)=0 \quad \text { for } \quad i \neq j .
\end{aligned}
$$

and is given by

$$
g=\frac{1}{z^{2}}\{d x \otimes d x+d y \otimes d y+d z \otimes d z\} .
$$

Also, let $\eta, \varphi$ be the 1 - form and the $(1,1)$-tensor field, respectively defined by

$$
\eta\left(Z, e_{3}\right)=1, \quad \varphi\left(e_{1}\right)=-e_{2}, \quad \varphi\left(e_{2}\right)=e_{1}, \quad \varphi\left(e_{3}\right)=0
$$

for any $Z \in \Gamma(T \tilde{M})$. Hence, $(\tilde{M}, \varphi, \xi, \eta, g)$ becomes an almost contact metric manifold with the characteristic vector field $e_{3}=\xi$.

By direct calculations, we have

$$
\left[e_{1}, e_{2}\right]=0, \quad\left[e_{1}, e_{3}\right]=e_{1} \quad \text { and }\left[e_{2}, e_{3}\right]=e_{2} .
$$

On the other hand, using Koszul's formula for the Riemannian metric $g$, we have:

$$
\tilde{\nabla}_{e_{1}} e_{3}=e_{1}, \quad \tilde{\nabla}_{e_{2}} e_{3}=e_{2}, \quad \tilde{\nabla}_{e_{3}} e_{3}=0
$$


and others

$$
\tilde{\nabla}_{e_{1}} e_{2}=\tilde{\nabla}_{e_{2}} e_{1}=\tilde{\nabla}_{e_{3}} e_{1}=\tilde{\nabla}_{e_{3} e_{2}}=0, \quad \tilde{\nabla}_{e_{1}} e_{1}=\tilde{\nabla}_{e_{2}} e_{2}=-e_{3} .
$$

Therefore, the manifold $\tilde{M}$ is a 3 -dimensional Kenmotsu manifold. Now, we suppose that $e_{3}=\xi$ is a torqued vector field on $\tilde{M}$. Then,

$$
\begin{aligned}
& \tilde{\nabla}_{e_{1}} \xi=f e_{1}+\alpha\left(e_{1}\right) \xi \quad \text { and } \quad \alpha(\xi)=0 \\
& \tilde{\nabla}_{e_{2}} \xi=f e_{2}+\alpha\left(e_{2}\right) \xi \quad \text { and } \quad \alpha(\xi)=0 \\
& \tilde{\nabla}_{e_{3}} \xi=f e_{3}+\alpha\left(e_{3}\right) \xi \quad \text { and } \quad \alpha(\xi)=0 \text {. }
\end{aligned}
$$

are satisfied. From (3.6), (3.8), (3.9) and (3.10), we get

$$
f=1 \quad \text { and } \quad \alpha\left(e_{3}\right)=\alpha(\xi)=-1 \neq 0,
$$

which is a contradiction. Therefore, $e_{3}=\xi$ is never torqued vector field on Kenmotsu manifold $\tilde{M}$.

Considering Theorem 3.1, we get the following:

Remark 3.3. Let $M$ be a submanifold endowed with a torqued vector field $\mathcal{T}$ of a Kenmotsu manifold $\tilde{M}$. Then, the characteristic vector field $\xi$ is never torqued on $M$.

Next, we have the following theorem.

Theorem 3.4. Let $M$ be a submanifold of a Kenmotsu manifold $\tilde{M}$ endowed with a torqued vector field $\mathcal{T}$. The submanifold $M$ is totally geodesic if and only if the tangential component $\mathcal{T}^{\top}$ of $\mathcal{T}$ is a torse-forming vector field on $M$ whose conformal scalar is the restriction of the torqued function and whose generating form is the restriction of the torqued function of $\mathcal{T}$ on $M$.

Proof. Since $\mathcal{T}$ is a torqued vector field on the ambient space $\tilde{M}$, it follows from (1.2), (2.14) and the formulas of Gauss and Weingarten, one has

$$
\nabla_{X} \mathcal{T}^{\top}+h\left(X, \mathcal{T}^{\top}\right)-A_{\mathcal{T} \perp} X+\nabla_{X}^{\perp} \mathcal{T}^{\perp}=f X+\alpha(X) \mathcal{T}^{\top}+\alpha(X) \mathcal{T}^{\perp}
$$

where $\nabla$ stands for the Levi-Civita connection on $M$, for any $X \in \Gamma(T M)$. By comparing the tangential and normal components of (3.12), we get

$$
\begin{aligned}
& h\left(X, \mathcal{T}^{\top}\right)+\nabla_{X}^{\perp} \mathcal{T}^{\perp}=\alpha(X) \mathcal{T}^{\perp}, \\
& \nabla_{X} \mathcal{T}^{\top}-A_{\mathcal{T} \perp} X=f X+\alpha(X) \mathcal{T}^{\top} .
\end{aligned}
$$

If $M$ is a totally geodesic submanifold of $\tilde{M}$, then the equation (3.13) becomes

$$
\nabla_{X} \mathcal{T}^{\top}=f X+\alpha(X) \mathcal{T}^{\top}
$$

which implies that $\mathcal{T}^{\top}$ is a torse-forming on $M$. The proof of the converse part is straightforward.

Considering the equality (3.13), we have the following cases:

From now on, we suppose that the submanifold $M$ admits a Ricci soliton in Theorem 3.4.

Case I: If we take $\mathcal{T}^{\top} \in \Gamma(D)$, then from (2.4), (2.9), (2.10) and (3.13) we get

$$
g\left(\nabla_{X} \mathcal{T}^{\top}, \xi\right)=g(f X, \xi),
$$

where $T M=D \oplus \operatorname{Span}\{\xi\}$, for any $X \in \Gamma(T M)$. Since the Riemannian metric $g$ is non-degenere, we have

$$
\nabla_{X} \mathcal{T}^{\top}=f X,
$$

which shows that the vector field $\mathcal{T}^{\top}$ is a concircular on $M$. 
On the other hand, from the definition of Lie-derivative and (3.16) one has

$$
\begin{aligned}
\left(£_{\mathcal{T}^{\top}} g\right)(X, Y) & =g\left(\nabla_{X} \mathcal{T}^{\top}, Y\right)+g\left(\nabla_{Y} \mathcal{T}^{\top}, X\right) \\
& =2 f g(X, Y)
\end{aligned}
$$

for any $X, Y \in \Gamma(T M)$, which means that the vector field $\mathcal{T}^{\top}$ is a conformal Killing. Also, from (1.1) and (3.17), we obtain

$$
S(X, Y)=-(\lambda+f) g(X, Y),
$$

where $S$ is the Ricci tensor of $M$. Hence, $M$ is an Einstein.

Case II: If we take $\mathcal{T}^{\top} \in \Gamma(D)$, then it follows from (3.15), we have

$$
g\left(\nabla_{X} \mathcal{T}^{\top}, \xi\right)=0
$$

for any $X \in \Gamma(D)$. As a consequence of the equation (3.18), $\mathcal{T}^{\top}$ is a parallel vector field on distribution $D$ and thus, $\mathcal{T}^{\top}$ is a $D$-Killing vector field.

On the other side, using (1.1) and (3.18) the Ricci tensor $S^{D}$ of the distribution $D$

$$
S^{D}(X, Y)=-\lambda g(X, Y)
$$

is found. Therefore, the distribution $D$ is an Einstein.

Case III: If we use $\xi$ instead of $\mathcal{T}^{\top}$ in (3.14), we have

$$
\nabla_{X} \xi=f X+\alpha(X) \xi
$$

for any $X \in \Gamma(T M)$. Taking the inner product of (3.19) with $\xi$, we get

$$
g\left(\nabla_{X} \xi, \xi\right)=f \eta(X)+\alpha(X)
$$

which yields

$$
\alpha(X)=-f \eta(X) .
$$

It is easy to see that $\alpha(\xi) \neq 0$. So, $\xi$ is a torse-forming on $M$.

Using the equality (3.13), we have the following:

Corollary 3.5. Let $M$ be a submanifold of a Kenmotsu manifold $\tilde{M}$ endowed with a torqued vector field $\mathcal{T}$. If $M$ is $\mathcal{T}^{\perp}$-umbilical, then $\mathcal{T}^{\top}$ is a torse-forming on $M$.

The next theorem gives a characterization as follows:

Theorem 3.6. Let $\tilde{M}$ be a Kenmotsu manifold endowed with a torqued vector field $\mathcal{T}$ and $M$ be a submanifold admitting a Ricci soliton of $\tilde{M}$. Then, $(M, g, \xi, \lambda)$ is an $\eta$-Einstein.

Proof. If we take $\xi$ instead of $\mathcal{T}^{\top}$ in (3.13), we have

$$
\nabla_{X} \xi-A_{\mathcal{T} \perp} X=f X+\alpha(X) \xi
$$

From the equalities (2.4), (2.6) and (3.20), we get

$$
A_{\mathcal{T} \perp} X=(1-f) X-(\eta(X)+\alpha(X)) \xi .
$$

Also, if we use the relations (2.1), (2.10) and (3.21), one has

$$
g\left(h(X, Y), \mathcal{T}^{\perp}\right)=(1-f) g(X, Y)-(\eta(X)+\alpha(X)) \eta(Y) .
$$

Interchanging the roles of $X$ and $Y$ in (3.22) gives

$$
g\left(h(Y, X), \mathcal{T}^{\perp}\right)=(1-f) g(Y, X)-(\eta(Y)+\alpha(Y)) \eta(X) .
$$

Since $h$ and $g$ are symmetric, from (3.22) and (3.23) we have

$$
\begin{aligned}
2 g\left(h(X, Y), \mathcal{T}^{\perp}\right)= & 2(1-f) g(X, Y)-2 \eta(X) \eta(Y) \\
& -\alpha(X) \eta(Y)-\alpha(Y) \eta(X)
\end{aligned}
$$

for any $X, Y \in \Gamma(T M)$. 
On the other hand, from the definition of Lie-derivative and (2.1), (2.10), (3.20) and (3.24), we obtain

$$
\begin{aligned}
\left(£_{\xi} g\right)(X, Y)= & g\left(\nabla_{X} \xi, Y\right)+g\left(\nabla_{Y} \xi, X\right) \\
= & g\left(f X+\alpha(X) \xi+A_{\mathcal{T}^{\perp}} X, Y\right) \\
& +g\left(f Y+\alpha(Y) \xi+A_{\mathcal{T}^{\perp}} Y, X\right) \\
= & 2 g(X, Y)-2 \eta(X) \eta(Y) .
\end{aligned}
$$

Since $M$ is a submanifold admitting a Ricci soliton and from the equalities (1.1) and (3.25), the Ricci tensor $S$ of $M$

$$
S(X, Y)=-(\lambda+1) g(X, Y)+\eta(X) \eta(Y)
$$

is satisfied. This means $M$ is an $\eta$-Einstein.

As a consequence of Theorem 3.6, we can state the followings:

Corollary 3.7. Let $\tilde{M}$ be a Kenmotsu manifold endowed with a torqued vector field $\mathcal{T}$ and $M$ be a submanifold admitting a Ricci soliton as its potential field $\xi$ of $\tilde{M}$. Then, $M$ has $\eta$-parallel Ricci tensor.

Corollary 3.8. Let $\tilde{M}$ be a Kenmotsu manifold endowed with a torqued vector field $\mathcal{T}$ and $M$ be a n-dimensional submanifold admitting a Ricci soliton as its potential field $\xi$ of $\tilde{M}$. Then, $M$ has constant scalar curvature $r$ given by

$$
r=1-n(\lambda+1)
$$

\section{Ricci solitons in Kenmotsu space form with torqued vector field}

In this section, we investigate the submanifolds admitting a Ricci soliton of Kenmotsu space form $\tilde{M}(c)$ endowed with torqued vector field $\mathcal{T}$.

Now, we are ready to give the next theorem as follows:

Theorem 4.1. Let $\tilde{M}(c)$ be a Kenmotsu space form and $M$ be a n-dimensional submanifold of $\tilde{M}(c)$. If $M$ is totally umbilical and the mean curvature $\|H\|$ is constant, then $M$ is $\eta$-Einstein.

Proof. Let $\left\{e_{1}, e_{2}, \ldots, e_{n-1}, \xi\right\}$ be an orthonormal basis of $T_{p} M, p \in M$. From the definition of the Ricci tensor, we have

$$
S(Y, Z)=\sum_{i=1}^{n-1} g\left(R\left(e_{i}, Y\right) Z, e_{i}\right)+g(R(\xi, Y) Z, \xi)
$$

where $R$ is the Riemann curvature tensor of the submanifold $M$. 
If we put $X=W=e_{i}$ in (2.11) and use the equalities (2.1), (2.2), (2.5), (2.9) and (2.12), then one has

$$
\begin{aligned}
\sum_{i=1}^{n-1} g\left(R\left(e_{i}, Y\right) Z,, e_{i}\right)= & \sum_{i=1}^{n-1} g\left(\tilde{R}\left(e_{i}, Y\right) Z, e_{i}\right)-g\left(h\left(e_{i}, e_{i}\right), h(Y, Z)\right) \\
& +g\left(h\left(e_{i}, Z\right), h\left(Y, e_{i}\right)\right) \\
= & \sum_{i=1}^{n-1} \frac{c-3}{4}\left\{g(Y, Z) g\left(e_{i}, e_{i}\right)-g\left(e_{i}, Z\right) g\left(Y, e_{i}\right)\right\} \\
& +\frac{c+1}{4}\left\{3 g\left(e_{i}, \varphi Y\right) g\left(\varphi Z, e_{i}\right)-\eta(Y) \eta(Z) g\left(e_{i}, e_{i}\right)\right\} \\
& +\sum_{i=1}^{n-1}\left(g\left(e_{i}, Z\right) g\left(Y, e_{i}\right)-\left(g\left(e_{i}, e_{i}\right) g(Y, Z)\right)\|H\|^{2}\right. \\
= & \frac{c-3}{4}\{(n-2) g(Y, Z)+\eta(Y) \eta(Z)\} \\
& +\frac{c+1}{4}\{3 g(Y, Z)-(n+2) \eta(Y) \eta(Z)\} \\
& +((n-2) g(Y, Z))+\eta(Y) \eta(Z))\|H\|^{2} .
\end{aligned}
$$

Similarly, taking $X=W=\xi$ in (2.11), we get

$$
g(R(\xi, Y) Z, \xi)=g(\tilde{R}(\xi, Y) Z, \xi)=\eta(Y) \eta(Z)-g(Y, Z)
$$

for any $Y, Z \in \Gamma(T M)$. Then, using (4.2) and (4.3) in (4.1), the Ricci tensor $S$ of $M$

$$
\begin{aligned}
S(Y, Z)= & \left(\frac{c(n+1)-3 n+5}{4}+(n-2)\|H\|^{2}\right) g(Y, Z) \\
& -\left(\frac{c(n+1)+n+1}{4}-\|H\|^{2}\right) \eta(Y) \eta(Z)
\end{aligned}
$$

is obtained which means that $M$ is an $\eta$-Einstein. This completes the proof.

Theorem 4.2. Let $\tilde{M}(c)$ be a Kenmotsu space form endowed with a torqued vector field $\mathcal{T}$ and $M$ be an $n$-dimensional $(n>1)$ totally umbilical submanifold admitting a Ricci soliton of $\tilde{M}$. Then, $M$ has a constant mean curvature.

Proof. If we put $Y=Z=\xi$ in (3.26) and using (2.1) and (2.2), we get

$$
S(\xi, \xi)=-\lambda \text {. }
$$

Similarly, if we take $Y=Z=\xi$ in (4.4) and also using (2.1) and (2.2), then we have

$$
S(\xi, \xi)=(1-n)\left(1-\|H\|^{2}\right) .
$$

Since $M$ is a Ricci soliton, from the equalities (4.5) and (4.6),

$$
\|H\|^{2}=1-\frac{\lambda}{n-1}
$$

is obtained which completes the proof of the theorem.

Using the equality (4.7), we can state the following corollary:

Corollary 4.3. Let $\tilde{M}(c)$ be a Kenmotsu space form endowed with a torqued vector field $\mathcal{T}$ and $M$ be an $n$-dimensional $(n>1)$ totally umbilical submanifold admitting a Ricci soliton of $\tilde{M}$. Then, we have the following:

i) If $\|H\|<1$, then the Ricci soliton $(M, g, \xi, \lambda)$ is expanding.

ii) If $\|H\|>1$, then the Ricci soliton $(M, g, \xi, \lambda)$ is shrinking.

iii) The Ricci soliton $(M, g, \xi, \lambda)$ is steady if and only if $\|H\|=1$. 


\section{Conclusion}

Ricci soliton is a natural generalization of Einstein manifold. This notion corresponds to the self-similar solution of Hamilton's Ricci flow. Over the last decades, the geometry of Ricci solitons has been studied by many mathematicians. In 2008, Sharma applied Ricci solitons to $K$-contact manifolds and launched the study of Ricci solitons. Since then, Ricci solitons have been studied. In this paper, we deal with the submanifold admitting a Ricci soliton of a Kenmotsu manifold endowed with torqued vector field $\mathcal{T}$. We find that the characteristic vector field $\xi$ is never torqued on submanifold $M$ of Kenmotsu manifold $\tilde{M}$. We obtain a necessary and sufficient condition for the tangential part $\mathcal{T}^{\top}$ of $\mathcal{T}$ to be a torse-forming on $M$. Also, we prove that if $M$ admits a Ricci soliton, then it is an $\eta$-Einstein. Finally, we study the submanifold $M$ admitting a Ricci soliton of a Kenmotsu space form $\tilde{M}(c)$ endowed with a torqued vector field $\mathcal{T}$ and obtain that if $M$ admits a Ricci soliton as its potential field $\xi$, then it is an expanding.

\section{References}

[1] C. S. Bagewadi, and G. Ingalahalli, Ricci Solitons in Lorentzian $\alpha$-Sasakian Manifolds, Acta Math. Acad. Paedagog. Nyházi. (N.S), 28 (1), 59-68, 2012.

[2] C. L. Bejan and M. Crasmareanu, Second Order Parallel Tensors and Ricci Solitons in 3-Dimensional Normal Paracontact Geometry, Ann. Glob. Anal. Geom., 46 , 117$127,2014$.

[3] D. E. Blair, Contact Manifolds in Riemannian Geometry, Lecture Notes in Mathematics, 509, Springer-Verlag, Berlin, 1976.

[4] B.-Y. Chen, Geometry of Submanifolds, Marcel Dekker, New York, 1973.

[5] B.-Y. Chen, Some Results on Concircular Vector Fields and Their Applications to Ricci Solitons, Bull. Korean Math. Soc., 52 (5), 1535-1547, 2015.

[6] B.-Y. Chen, Rectifying Submanifolds of Riemannian Manifolds and Torqued Vector Fields, Kragujevac J. Math., 41 (1), 93-103, 2017.

[7] B.-Y. Chen, Classification of Torqued Vector Fields and Its Applications to Ricci Solitons, Kragujevac J. Math., 41 (2), 239-250, 2017.

[8] J. T. Cho and J. Park, Gradient Ricci Solitons with Semi-Symmetry, Bull. Korean Math. Soc., 51 (1), 213-219, 2014.

[9] A. Ghosh, Certain Contact Metrics as Ricci Almost Solitons, Results Maths., 65, 81-94, 2014.

[10] A. Ghosh, Kenmotsu 3-Metric as a Ricci Soliton, Chaos, Solitons \& Fractals, 44 (8), 647-650, 2011.

[11] R. S. Hamilton, Three-Manifolds with Positive Ricci Curvature, J. Diff. Geom., 17 (2), 255-306, 1982.

[12] R. S. Hamilton, The Ricci Flow on Surfaces, Mathematics and General Relativity (Santa Cruz, CA, 1986), Contemp. Math., A.M.S, 71, 237-262, 1988.

[13] S. K. Hui, S. K. Yadav and A. Patra, Almost Conformal Ricci Solitons on f-Kenmotsu Manifolds, Khayyam J. Math., 5 (1), 89-104, 2019.

[14] J.-B. Jun, U. C. De and G. Pathak, On Kenmotsu Manifolds, J. Korean Math. Soc., 42 (3), 435-445, 2005.

[15] K. Kenmotsu, A Class of Almost Contact Riemannian Manifolds, Tohoku Math. J., 24, 93-103, 1972.

[16] H. G. Nagaraja and C. R. Premalatha, Ricci Solitons in Kenmotsu Manifolds, J. Math. Anal., 3 (2), 18-24, 2012.

[17] S. Y. Perktaş and S. Keleş, Ricci Solitons in 3-Dimensional Normal Almost Paracontact Metric Manifolds, Int. Electron. J. Geom., 8 (2), 34-45, 2015.

[18] R. Sharma, Certain Results on K-Contact and $(k, \mu)$-Contact Manifolds, J. Geom., 89, (1-2), 138-147, 2008. 
[19] R. Sharma and A. Ghosh, Sasakian 3-Manifolds as a Ricci Soliton Represents the Heisenberg Group, Int. J. Geom. Methods Mod. Phys, 8 (1), 149-154., 2011.

[20] S. Sular and C. Özgür, On Some Submanifolds of Kenmotsu Manifolds, Chaos, Solitons \& Fractals, 4 (2), 1990-1995, 2009.

[21] M. M. Tripathi, Ricci Solitons in Contact Metric Manifolds, arXiv:0801.4222v1, [math DG], 2008.

[22] K. Yano and M. Kon, Structures on Manifolds, Series in Mathematics, World Scientific Publishing, Springer, 1984.

[23] H. İ. Yoldaş, Ş. E. Meriç, E. Yaşar, On Generic Submanifold of Sasakian Manifold with Concurrent Vector Field, Commun. Fac. Sci. Univ. Ank. Ser. A1 Math. Stat., 68 (2), 1983-1994, 2019. 